\title{
Synthesis and Magnetic Properties of Ni and Carbon Coated Ni by Levitational Gas Condensation (LGC)
}

\author{
Young Rang Uhm ${ }^{1}$ and Chang Kyu Rhee ${ }^{2}$ \\ ${ }^{1}$ Radioisotope Research Division, Korea Atomic Energy Research Institute, 989 Daedukdaero, Daejeon 305-353, Republic of Korea \\ ${ }^{2}$ Nuclear Materials Development Research Division, Korea Atomic Energy Research Institute, 989 Daedukdaero, \\ Daejeon 305-353, Republic of Korea
}

Correspondence should be addressed to Young Rang Uhm; uyrang@kaeri.re.kr

Received 6 March 2013; Revised 21 June 2013; Accepted 22 June 2013

Academic Editor: Takeshi Seki

Copyright (c) 2013 Y. R. Uhm and C. K. Rhee. This is an open access article distributed under the Creative Commons Attribution License, which permits unrestricted use, distribution, and reproduction in any medium, provided the original work is properly cited.

The nickel (Ni), and carbon coated nickel (Ni@C) nanoparticles were synthesized by levitaional gas condensation (LGC) methods using a micron powder feeding (MPF) system. Both metal and carbon coated metal nano powders include a magnetic ordered phase. The synthesis by LGC yields spherical particles with a large coercivity. The abnormal initial magnetization curve for Ni indicates a non-collinear magnetic structure between the core and surface layer of the particles. The carbon coated particles had a core structure diameter at and below $10 \mathrm{~nm}$ and were covered by 2-3 nm thin carbon layers. The hysteresis loop of the as-prepared $\mathrm{Ni@Cs} \mathrm{materials} \mathrm{with} \mathrm{unsaturated} \mathrm{magnetization} \mathrm{shows} \mathrm{a} \mathrm{superparamagnetic} \mathrm{state} \mathrm{at} \mathrm{room} \mathrm{temperature.}$

\section{Introduction}

Nanoparticles have achieved significant attention owing to the novel electro-optic, magnetic, and catalytic properties that arise from the quantum size effect and large specific surface areas that are characteristic of nanosized species $[1,2]$. Magnetic nanoparticles have attracted much of the attention these days, because of their potential technological applications in nanofluids for biomedical application, thermal conductive fluids, various catalysts, and so forth [1-3]. However, the preparation of nanofluids using pure metal nanoparticles is very hard, because nanoparticles are inherently vulnerable to oxidation, dissolution and agglomeration. In particular, agglomeration of the particles in a solvent is a serious problem when preparing nanofluids. To overcome these problems, a protective shell has been recommended to improve the chemical stability of metal nanoparticles and the dispersion stability in the solvent [46 ]. It is also worth noting that these materials are not prone to agglomeration owing to the fact that the addition of a carbon coating layer reduces the magnetic interaction. In addition, the surface diffusion processes allow the preservation of the chemical and structural properties of the nanopowder for a long time in many chemically aggressive surroundings [7]. In particular, a graphitic carbon shell is regarded as an ideal coating as it is light and shows high stability in both chemical and physical environments $[8,9]$. The stable dispersion of nanoparticles in both aqueous and nonaqueous media has important applications in cosmetics, chemicals, pharmaceuticals, microelectronics, paints, and pigments [10, 11]. Nanoparticle dispersion depends on many factors, such as the solvent, $\mathrm{pH}$, added ion, dispersant, and particle size. In particular, the preparation of a stable suspension of metallic nanoparticles is difficult owing to the high density and agglomeration of particles. Carbon-encapsulated metal nanoparticles are expected to have a large advantage for preventing the coalescence of the particles in the fluid. In this study, we introduce $\mathrm{Ni}$ and carbon-coated Ni (Ni@C) nanoparticles synthesized using LGC [11]. We also report on the dispersion stability in various solutions, such as water, ethanol, and ethylene glycol.

\section{Experiment}

High purity Ni and Ni@C nanopowders were synthesized by a LGC method using a micron powder feeding (MPF) system 

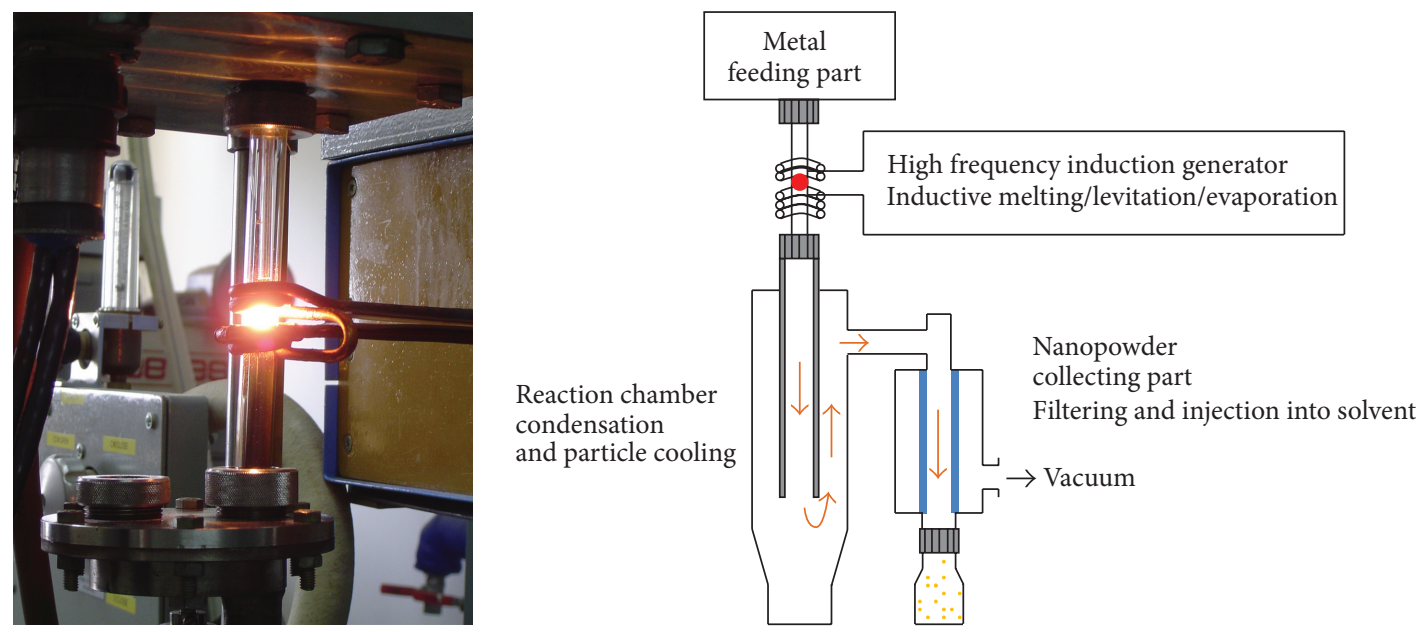

FIGURE 1: The shape of inductor and the concept of system for LGC.

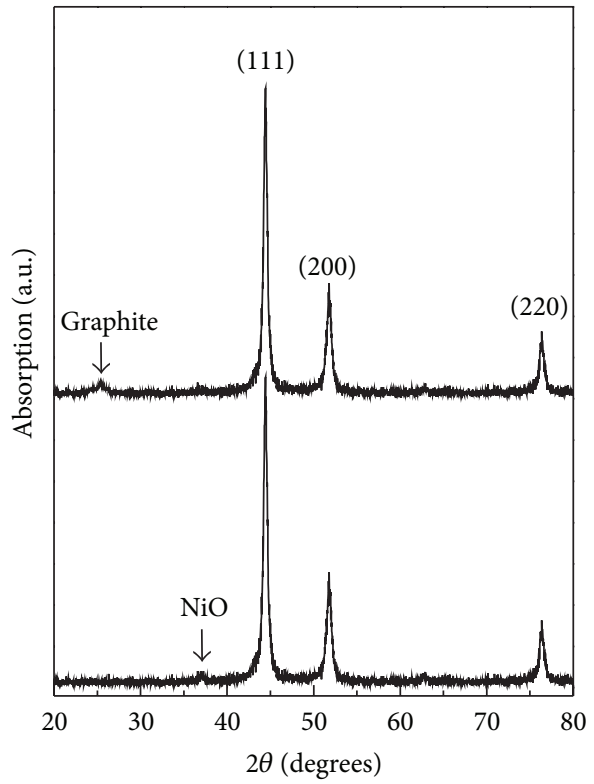

FIGURE 2: X-ray diffraction patterns for the $\mathrm{Ni}$ and $\mathrm{Ni@C} \mathrm{nanopar-}$ ticles.

[11]. The apparatus consists of a high-frequency induction generator of $6 \mathrm{~kW}$, a levitation and evaporation chamber, and a methane $\left(\mathrm{CH}_{4}\right)$ concentration control unit. Ni powder $(99.9$ at.\%, $\sim 500 \mu \mathrm{m})$ was used as a starting material for the synthesis of carbon encapsulated Ni nanoparticles. The $\mathrm{Ni}$ powders were mixed into the micron powder feeding system consisting of a rotation part for supplying the $\mathrm{Ni}$ micron powders to the melted droplet and a vibrating part for protecting the aggregation of the powder. The Ni micron powders were fed into a powder feeding system with the feeding rate of $80 \mathrm{mg} / \mathrm{min}$. A Ni ingot of $87 \mathrm{mg}$, as a seed material for levitation and evaporation reactions, was melted by electric induction heat. The gas pressure for the Ar in chamber was 100 torr. The ratio of $\mathrm{CH}_{4}$ was $10 \%$ of the mixture gas, when the carbon coated $\mathrm{Ni}$ was prepared.
The as-prepared sample was characterized by X-ray diffraction (XRD), transmission electron microscopy (TEM), and a vibrating sample magnetometer (VSM). The suspensions of $\mathrm{Ni@C} \mathrm{nanoparticles} \mathrm{were} \mathrm{prepared} \mathrm{as} \mathrm{follows:} \mathrm{we} \mathrm{prepared}$ solvents of ethanol and polyethylene glycol (MW 20000) in $100 \mathrm{~mL}$; we then added $0.01 \mathrm{wt} . \%$ of nanoparticles and ultrasonicated the suspension for $20 \mathrm{~min}$. To characterize the dispersion stability, we used Turbiscan Lab. and took measurements every 1 hour for 3 days.

\section{Results and Discussion}

The Ni and Ni@Cs materials were obtained using the LGC. A liquid droplet which is levitated by the magnetic force acted against the gravitational force from the coupled induction coils. The induction heat coil and the concept of LGC system are displayed at Figure 1. The inductor is heated up to $2000^{\circ} \mathrm{C}$ and the metallic atoms evaporated from the overheated surface of the liquid droplet and condensed by cold inert gas and then collected into the filter [11]. The mechanism for the formation of $\mathrm{Ni}$ nanoparticles is evaporation and condensation. At the same time, the molecular $\mathrm{CH}_{4}$ introduced into the chamber is converted into atomic $\mathrm{C}$ and $\mathrm{H}$ with high activity under high temperature. The highly active $\mathrm{C}$ atoms can react with $\mathrm{Ni}$ atoms. The $\mathrm{H}$ atoms converted into $\mathrm{H}_{2}$ molecules. The newly created $\mathrm{H}_{2}$ gas is vented out of the reaction chamber by continuous vacuum operation. The mechanism for the formation of graphitic carbon shell is reaction between active $\mathrm{C}$ atom and metal without chemical compounds of carbides [12].

Nanoparticles Ni and Ni@C synthesized by LGC using a micron powder feeding system were confirmed by an XRD pattern, as shown in Figure 2. The XRD results for Ni shows lattice parameters and the position of the main peaks of the $\mathrm{Ni}$ powders. The small amount of $\mathrm{NiO}$ phase was found in the XRD patterns and in the TEM images, which results from a passivation on the powder surface. The microstructure and phase composition of Ni powders were studied using TEM. A particle consists of a single domain. The powders with 


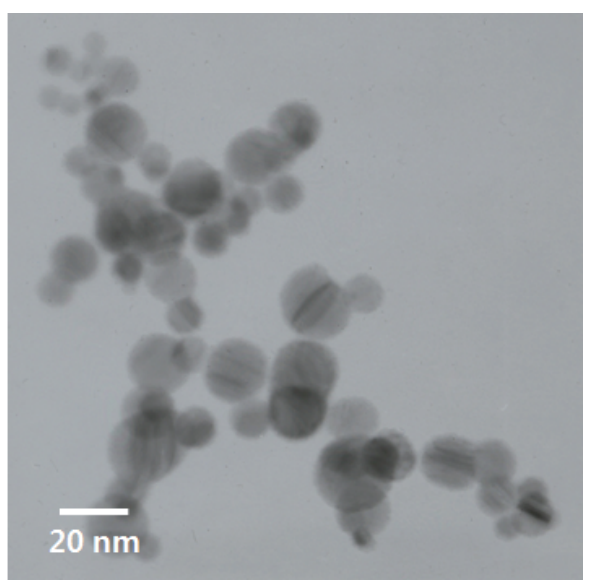

(a)

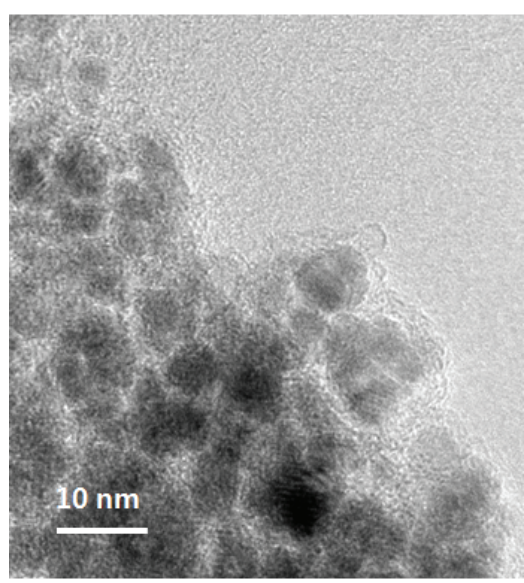

(b)

Figure 3: TEM images of (a) the Ni and (b) the Ni@C nanoparticles.

single domains are synthesized by a special gas phase method, though the average particle size is over $100 \mathrm{~nm}$ [12]. The thin oxide layer on powders forms a continuous coating with a thickness of 2-3 nm. Figure 2(b) shows the XRD patterns of the as-obtained Ni@C. All the main reflection peaks can be indexed to Ni. The strong nature of the peaks indicates that the final products are well crystallized and have high purity. The case of Ni@Cs materials is due to the (l 002 ) diffraction of graphite layers. However, the intensity for the $\left(\begin{array}{lll}0 & 0 & 2\end{array}\right)$ peak of the sample is broad and low. The shell layer in the Ni@Cs samples is made of amorphous carbon.For the Ni@Cs material, the diffraction peaks at $44.4^{\circ}, 51.8^{\circ}$, and $76.3^{\circ}$ are due to the (1 11 1), (2 00 ), and (2 20 ) planes of fcc-Ni, respectively.

The carbon shell structure of the Ni@Cs samples, which is further confirmed by the TEM results, is discussed in Figure 3. Nanoparticles Ni and Ni@C synthesized by LGC show a spherical shape as shown in Figure 3. The TEM image shows that nanocrystalline $\mathrm{Ni}$ powders consist of particles with the size ranges from $15 \mathrm{~nm}$ to $40 \mathrm{~nm}$. The magnetic properties of nanopowders were affected by the size effect resulting from the anisotropy field and magnetic domain effect on the particles [13]. The morphologies of the carboncoated $\mathrm{Ni}$ nanocrystallites were characterized by TEM as shown in Figure 3(b). The results indicated that all of the asmade materials were composed of only nanocapsules with a uniform particle size at and below $10 \mathrm{~nm}$. At the previous study, nanocapsules consisted of outer multishells of carbon were observed using a high resolution TEM [13].

The powders synthesized by LGC method show low saturation magnetization for $\mathrm{Ni}$. This results from a spin-canting effect and oxide phase on the surface [11]. The magnetic properties would be weak due to antiferromagnetic $\mathrm{NiO}$ phase on the powder surface. The saturation magnetization is $\mathrm{Ms}=42 \mathrm{emu} / \mathrm{g}$. In Figure 4(a), the hysteresis loop of $\mathrm{Ni}$ in the low fields is shown. The slightly shifted hysteresis loop for the Ni sample can be explained by exchange bias between the ferromagnetic core of $\mathrm{Ni}$ and antiferromagnetic surface of $\mathrm{NiO}[12,14]$. The initial magnetization curve is not explained by the size effect. At previous studies, the virgin magnetization curve slightly spills over the limited hysteresis loop at 655 Oe [13]. We assume that this effect enhances when the size of the particles is reduced, at previous study. With a decreasing particle size, the defects and the different magnetic structure on the surface of the particles are increased [13]. The nature of this irreversibility in high magnetic fields allows for the description of a physical model $[12,13]$. This irreversibility can be explained by a spin-glass or spin-canting behavior. The VSM magnetic measurement results for the synthesized Ni@Cs materials are shown in Figure 4(b). The hysteresis loop of the asmade M@Cs materials in the magnetic field up to $1 \mathrm{~T}$ reveals the intrinsic magnetic behaviour, indicated by the magnetization $(\mathrm{M})$, the remanent magnetization $(\mathrm{Mr})$ and the coercive force (Hc) for the M@Cs samples (Figure 4(b)). The saturation magnetization demonstrated that the carboncoated $\mathrm{Ni}$ nanocrystallites exhibited a superparamagnetic behaviour at room temperature. The magnetization was not saturated in the applied field up to $2 \mathrm{~T}$ as shown in Figure 4(c). The size of the nanoparticles may affect the value of the coercive field due to the size influence of the magnetization of the nanoparticles $[13,14]$. In the case of very small nanoparticles one can observe superparamagnetic behaviour, related to the demagnetization effect arising from the additional energy of the magnetic fields outside the graphitic carbon encapsulation. The coercive force $(\mathrm{Hc})$ and magnetization (M) were $76.6 \mathrm{Oe}$ and $19.6(\mathrm{emu} / \mathrm{g})$, respectively. The ratio of remanence to the saturation magnetization $(\mathrm{Mr} / \mathrm{M})$ of the $\mathrm{Ni@Cs} \mathrm{samples} \mathrm{is} \mathrm{0.04.} \mathrm{The} \mathrm{low} \mathrm{magnetization} \mathrm{comparing}$ with the Ni nanoparticles without carbon-shell results in the coexistence of nonmagnetic carbon and a large surface spin percentage with disordered magnetization orientation for the nanoparticles $[14,15]$. The magnetization and coercive force for Ni and Ni@C nanaoparticles were influenced by both the particle size and the surface status on the particle.

To evaluate the dispersion stability and agglomeration phenomena of the carbon encapsulated $\mathrm{Ni}$ nanoparticles in solvents of ethanol and polyethylene glycol (PEG), the timedependent sedimentation behavior was investigated by the 


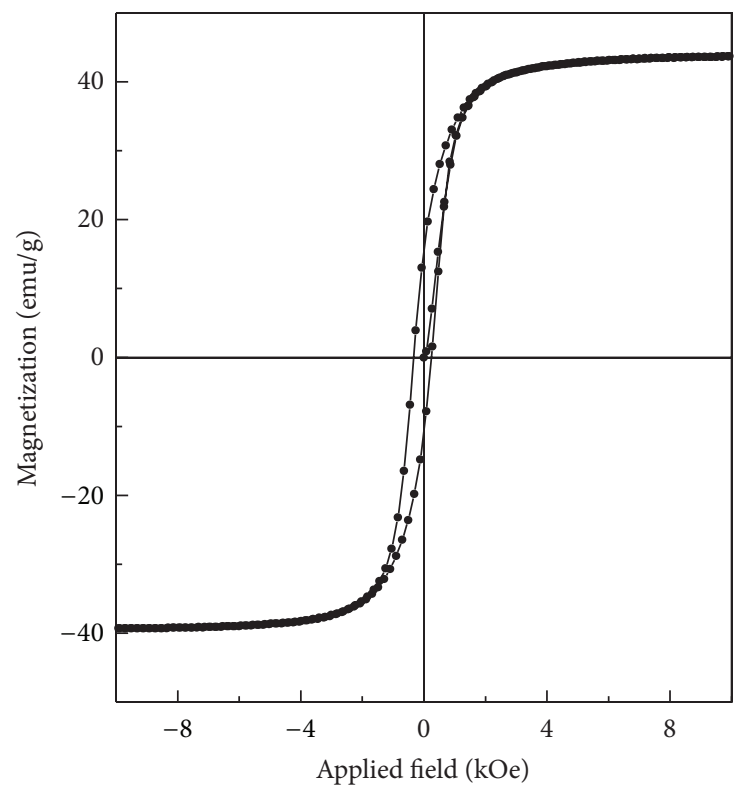

(a)

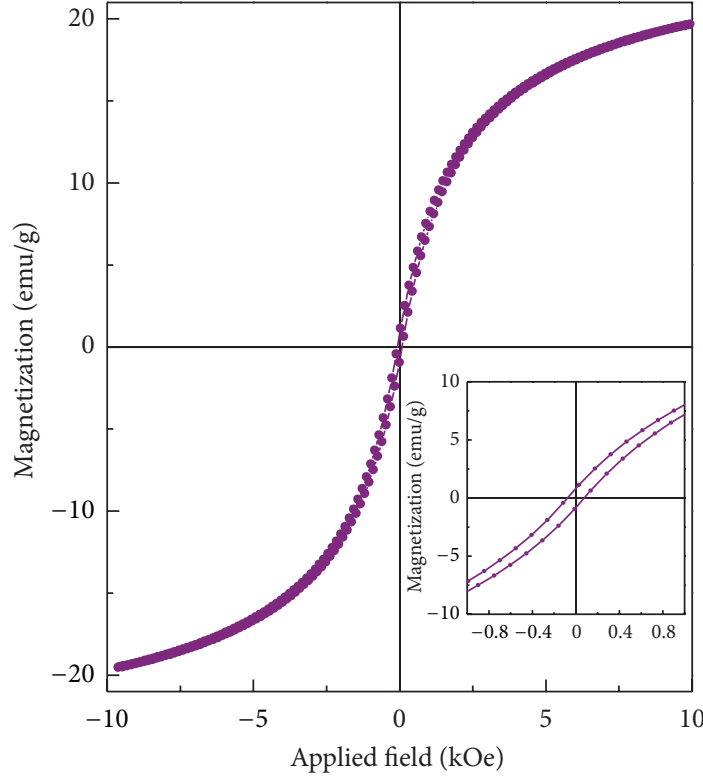

(b)

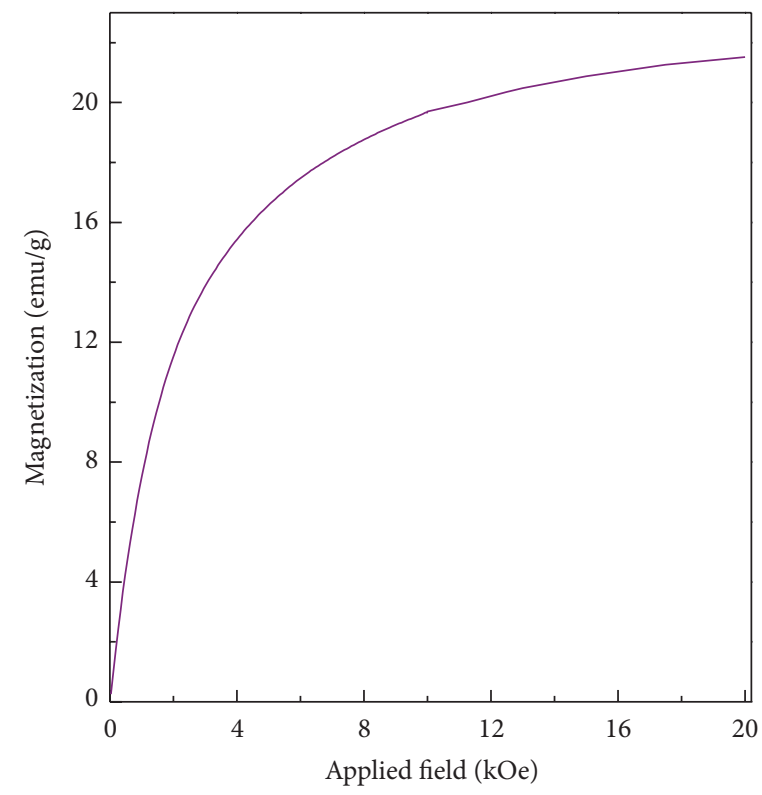

(c)

FIGURE 4: The hysteresis loops of (a) Ni and (b) Ni@C nanoparticles at room temperature. (Magnification of hysteresis loop for Ni@C is represented.) (c) Initial magnetization curve for Ni@C nanoparticles at room temperature.

transmission profile measurements using a Turbiscan Lab. The transmission profiles were taken every $1 \mathrm{hr}$ for $72 \mathrm{hrs}$ when the suspending medium was ethyl alcohol. It was found that the transmission intensity decreased at the sample top owing to a clarification and increased at the sample bottom from sedimentation, as shown in Figure 5 (a2) and (b2). A very stable Ni@C dispersion was observed without showing any clarification or sedimentation, as shown in Figure 5 (al) and (b1). On the contrary, a progressive fall of the signal, which was observed as a function of time in the middle region of the Ni nanoparticles with an average particle size of $20 \mathrm{~nm}$, can be explained by flocculation-induced particle growth as shown in Figure 5 (a2) and (b2).

\section{Conclusions}

Nanopaticles $\mathrm{Ni}$ and carbon coated $\mathrm{Ni}$ were prepared by LGC. A simple approach to fabricate a carbon-shell layer is to use methane $\left(\mathrm{CH}_{4}\right)$ gas as the carbon source. The particle size of carbon-coated $\mathrm{Ni}$ with diameters in the range of up to $10 \mathrm{~m}$ was smaller than those of $\mathrm{Ni}$ nanoparticles 


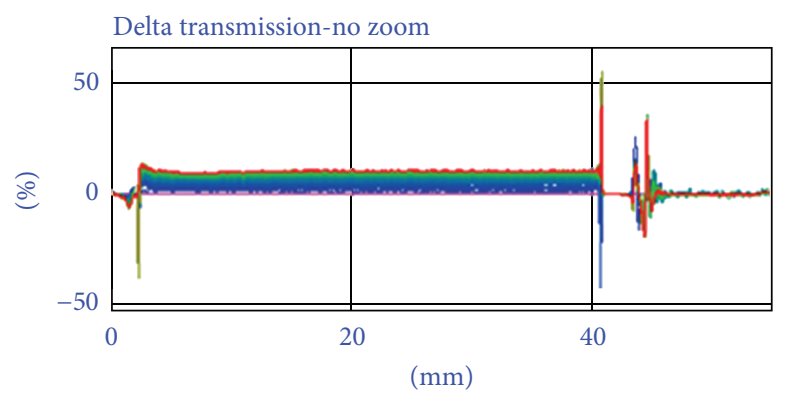

(a1)

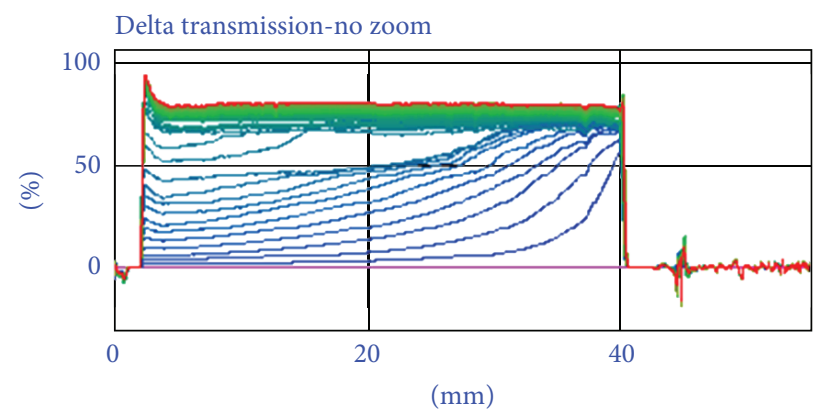

(a2)

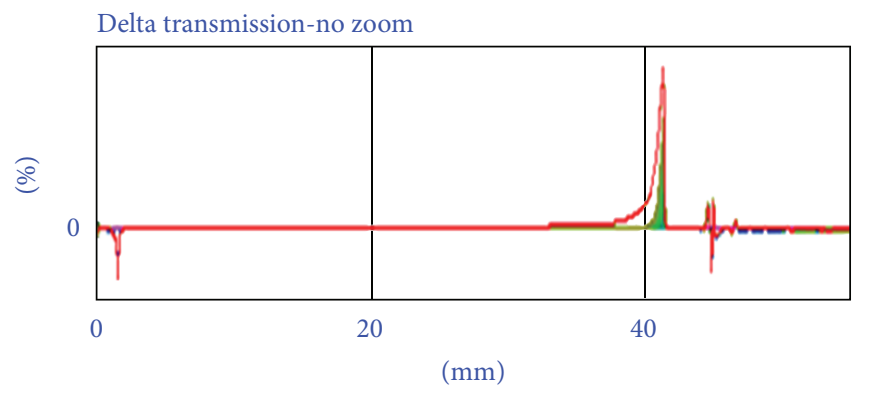

(b1)

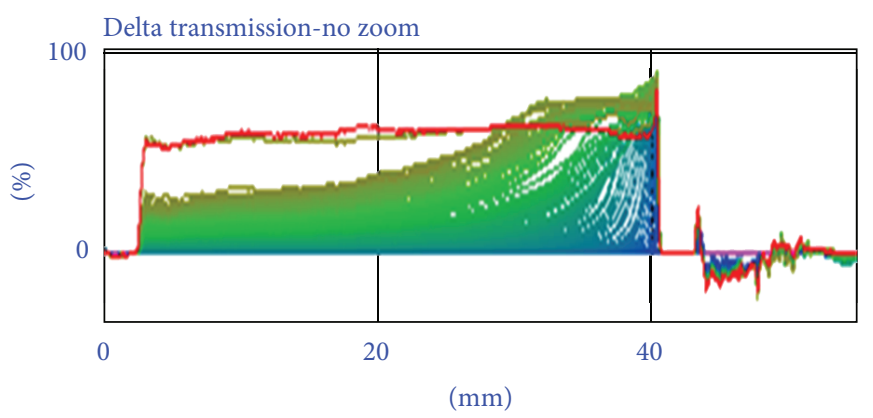

(b2)

Figure 5: Transmission profiles for (a1) Ni@C and (a2) Ni nanopowders in ethanol and (b1) Ni@C and (b2) Ni nanoparticles in polyethylene glycol (PEG) at $25^{\circ} \mathrm{C}$.

without a carbon shell. The dispersion stability kinetics of the PEG showed good dispersion. However, the sedimentation and flocculation behavior were observed in Ni nanoparticles without a carbon shell. The fully coated carbon shell layers of the Ni nanoparticles positively affected the stable dispersion in the fluid. Both the particle size and surface layer influence the magnetic hysteresis behavior of nanopowders.

\section{Acknowledgment}

This work was performed with financial support from the Industrial Source Technology Development Program (10043868) of the Ministry of Trade, Industry \& Energy, Korea.

\section{References}

[1] R. S. Ruoff, D. C. Lorents, B. Chan, R. Malhotra, and S. Subramoney, "Single crystal metals encapsulated in carbon nanoparticles," Science, vol. 259, no. 5093, pp. 346-348, 1993.

[2] J. H. J. Scott and S. A. Majetich, "Morphology, structure, and growth of nanoparticles produced in a carbon arc," Physical Review B, vol. 52, no. 17, pp. 12564-12571, 1995.

[3] Y. Kinemuchi, K. Ishizaka, H. Suematsu, W. Jiang, and K. Yatsui, "Magnetic properties of nanosize $\mathrm{NiFe}_{2} \mathrm{O}_{4}$ particles synthesized by pulsed wire discharge," Thin Solid Films, vol. 407, no. 1-2, pp. 109-113, 2002.

[4] J. Fang, N. Shama, L. Duc Tung et al., "Ultrafine $\mathrm{NiFe}_{2} \mathrm{O}_{4}$ powder fabricated from reverse microemulsion process," Journal of Applied Physics, vol. 93, no. 10, pp. 7483-7485, 2003.
[5] U. Jeong, X. Teng, Y. Wang, H. Yang, and Y. Xia, "Superparamagnetic colloids: controlled synthesis and niche applications," Advanced Materials, vol. 19, no. 1, pp. 33-60, 2007.

[6] S. Subramoney, "Novel nanocarbons-structure, properties, and potential applications," Advanced Materials, vol. 10, no. 15, pp. 1157-1171, 1998.

[7] B. H. Liu, J. Ding, Z. Y. Zhong, Z. L. Dong, T. White, and J. Y. Lin, "Large-scale preparation of carbon-encapsulated cobalt nanoparticles by the catalytic method," Chemical Physics Letters, vol. 358, no. 1-2, pp. 96-102, 2002.

[8] H. Caoa, G. Huangb, S. Xuana, Q. Wu, F. Gua, and C. Li, “Synthesis and characterization of carbon-coated iron core/shell nanostructures," Journal of Alloys and Compound, vol. 448, pp. 272-276, 2008.

[9] T. Hayashi, S. Hirono, M. Tomita, and S. Umemura, "Magnetic thin films of cobalt nanocrystals encapsulated in graphite-like carbon," Nature, vol. 381, no. 6585, pp. 772-774, 1996.

[10] S. H. Woo, M. K. Lee, and C. K. Rhee, "Sedimentation properties of $\mathrm{TiO}_{2}$ nanaoparticles in organic solvents," Solid State Phenomina, vol. 119, pp. 267-270, 2007.

[11] B. S. Han, C. K. Rhee, M. K. Lee, and Y. R. Uhm, "Synthesis of nano crystalline $\mathrm{Ni}$ and $\mathrm{Fe}$ by levitational gas condensation method," IEEE Transactions on Magnetics, vol. 42, no. 11, pp. 3779-3781, 2006.

[12] Y. R. Uhm, J. H. Park, W. W. Kim, C.-H. Cho, and C. K. Rhee, "Magnetic properties of nano-size Ni synthesized by the pulsed wire evaporation (PWE) method," Materials Science and Engineering B, vol. 106, no. 3, pp. 224-227, 2004.

[13] A. Y. Yermakov, M. A. Uimin, A. A. Mysik et al., "Magnetism and structure of nanoparticles and mesoscopic systems," Materials Science Forum, vol. 386-388, pp. 455-464, 2002. 
[14] B. D. Cullity, Introduction To Magnetic Materials, AddisonWesley, Reading, Mass, USA, 1972.

[15] C. Yu and J. S. Qiu, "Preparation and magnetic behavior of carbon-encapsulated cobalt and nickel nanoparticles from starch," Chemical Engineering Research and Design, vol. 86, no. 8, pp. 904-908, 2008. 

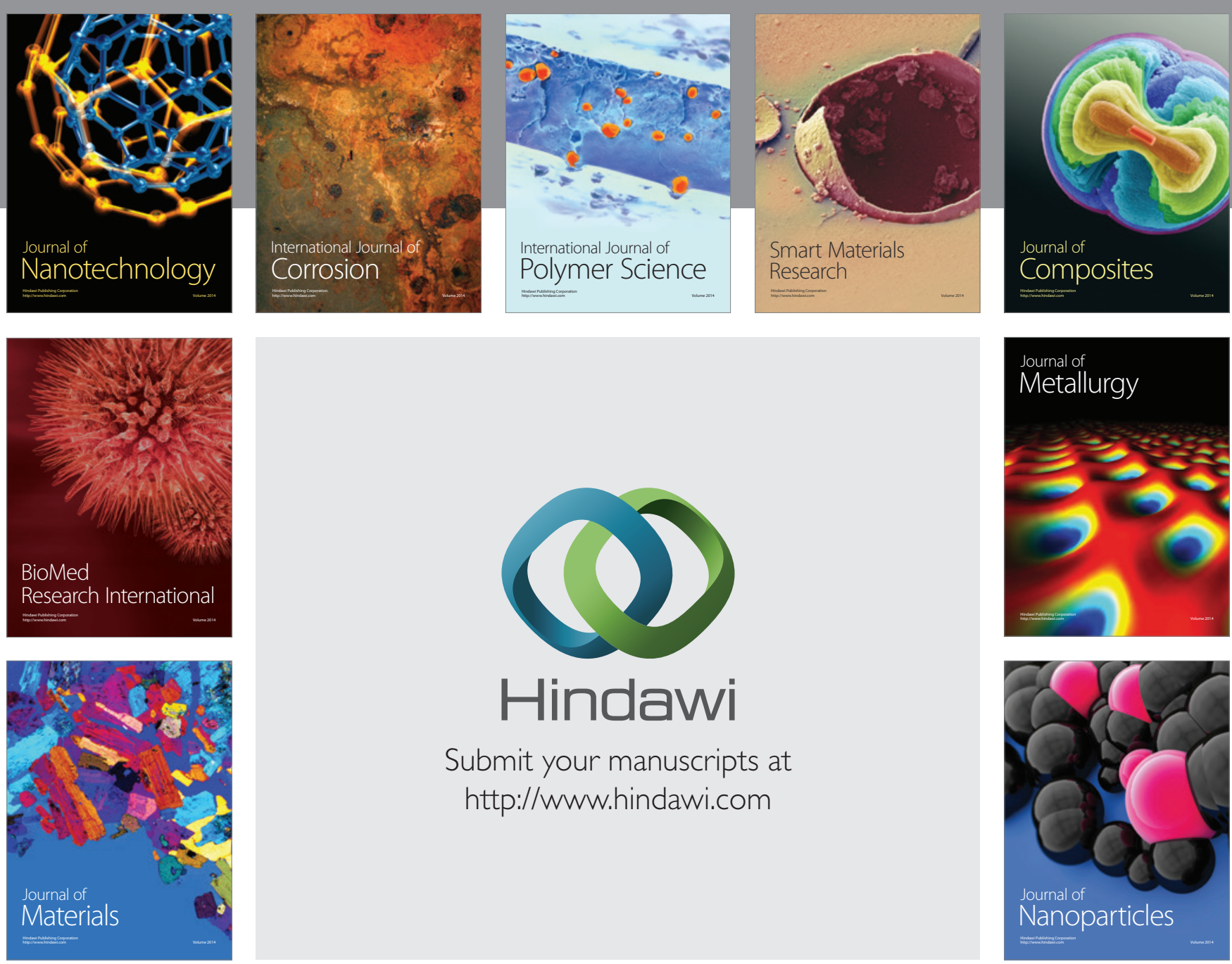

Submit your manuscripts at http://www.hindawi.com
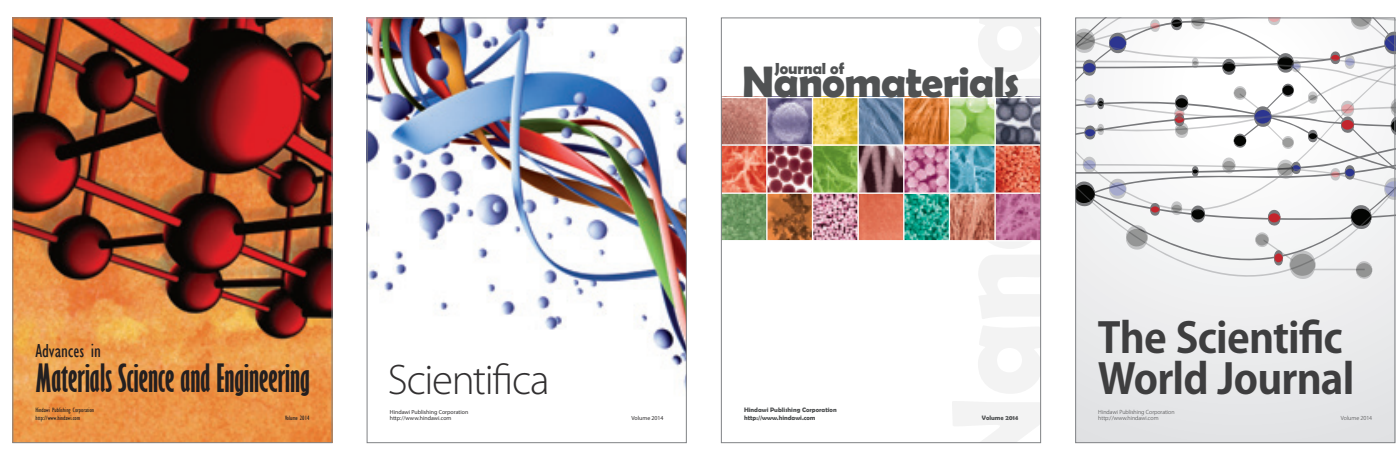

\section{The Scientific World Journal}
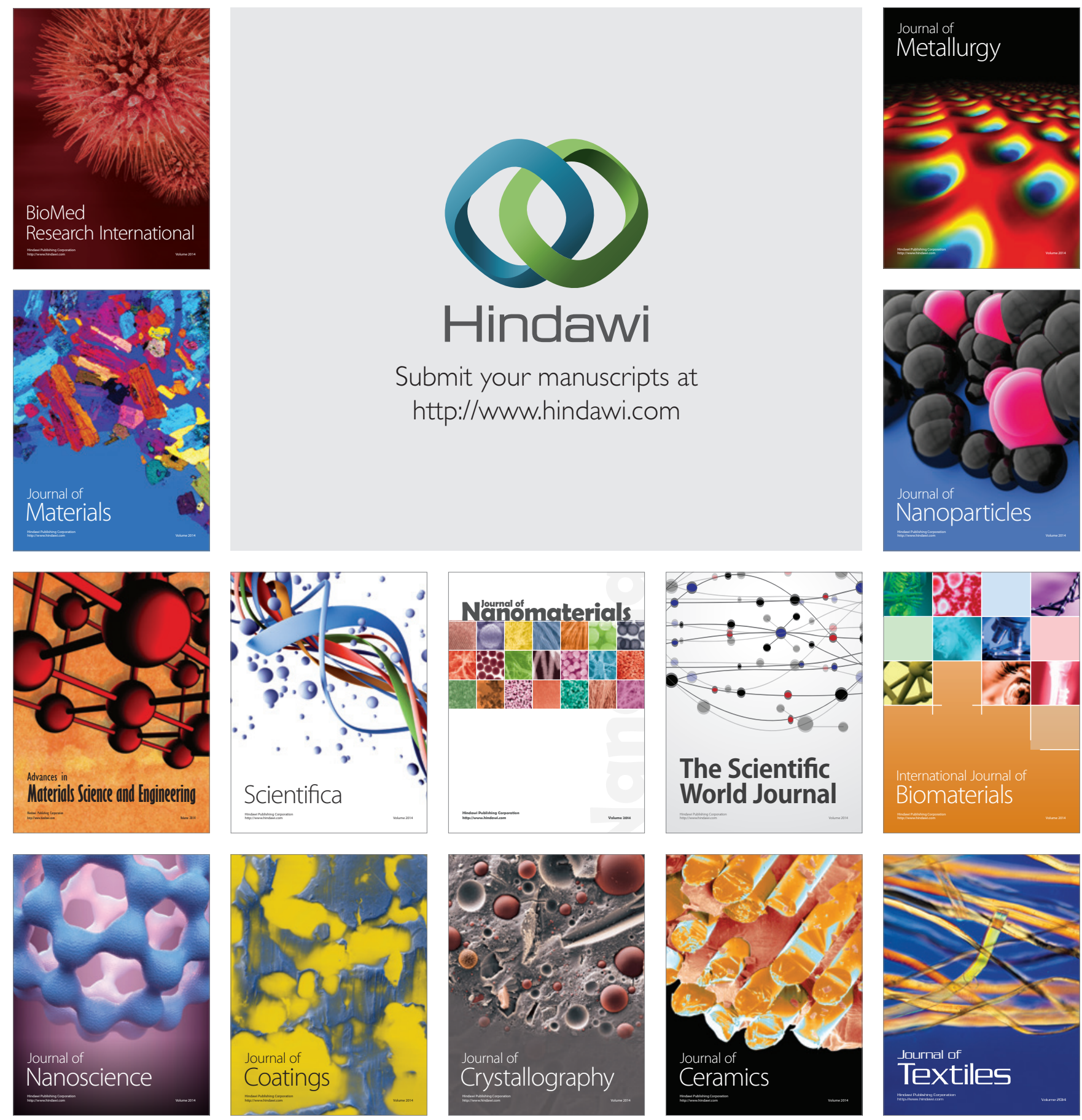